\title{
NON-CLASSICAL DIFFUSION EQUATIONS RELATED TO BIRTH-DEATH PROCESSES WITH TWO BOUNDARIES
}

\author{
BY \\ Z. G. MANSOURATI (Bell Northern Research Ltd., Ottawa, Ontario, Canada) \\ A.ND \\ L. L. CAMPBELL (Department of Mathematics and \\ Statistics, Queen's University, Kingston, Ontario, Canada)
}

\begin{abstract}
A pair of forward and backward diffusion equations is considered. In the forward equation, boundary values appear in the differential equation, and in the backward equation, boundary values are related to average values of the solution in the interior of the domain. The forward equation can be regarded as a diffusion approximation to a type of birth-death process with returns to the interior, or as a heat equation in one dimension where heat flowing out from the boundaries is returned to the interior. Existence and uniqueness theorems are proved, and some properties of the associated eigenvalues and eigenfunctions are deduced. An expression for the steady-state solution is obtained. Some information on the goodness of the diffusion approximation is also obtained.
\end{abstract}

1. Introduction. In [3], the second author studied a birth-death process with two boundaries. When the number of states becomes very large, the governing system of ordinary differential equations can be replaced by a single partial differential equation, the so-called diffusion approximation. For the equations in [3], the diffusion approximation takes the form

$$
\begin{aligned}
\frac{\partial \varphi}{\partial \tau}(x, \tau)= & \frac{\sigma^{2}}{2} \frac{\partial^{2} \varphi}{\partial x^{2}}(x, \tau)-\beta \frac{\partial \varphi}{\partial x}(x, \tau) \\
& +\frac{\sigma^{2}}{2}\left[F_{L}^{\prime}(x) \frac{\partial \varphi}{\partial x}(0, \tau)-F_{R}^{\prime}(x) \frac{\partial \varphi}{\partial x}(1, \tau)\right], \quad 0 \leq x \leq 1, \tau \geq 0,
\end{aligned}
$$

where $F_{L}^{\prime}$ and $F_{R}^{\prime}$ are derivatives of probability distribution functions and where the solution must satisfy the boundary conditions $\varphi(0, \tau)=\varphi(1, \tau)=0$. If $F_{L}$ or $F_{R}$ has discontinuities, the corresponding derivative in (1.1) gives rise to a discontinuity condition on $\partial \varphi / \partial x$ which is described more precisely in Sec. 2. Equation (1.1) could also describe

Received July 19, 1993.

1991 Mathematics Subject Classification. Primary 35K20; Secondary 60J80.

The research reported here was supported by the Natural Sciences and Engineering Research Council of Canada, Grant No. OGP0002151. 
a one-dimensional heat flow in which heat flowing out from the left and right boundaries is returned to the interior according to distribution functions $F_{L}$ and $F_{R}$ respectively.

The presence of the boundary values of $\partial \varphi / \partial x$ at $x=0$ and $x=1$ in (1.1) produces an equation of a type that seems not to have been considered in the literature. A special case of (1.1) was solved in [2]. The main object of this paper is to study the existence, uniqueness, and properties of solutions of (1.1) and its adjoint. Because both steadystate and time-dependent solutions are of interest in some applications, it is useful to examine the properties of the eigenvalues and eigenfunctions of the ordinary differential equation which is obtained from (1.1) by separating variables or by use of the Laplace transform. The convergence of the residue expansion that results from inversion of the Laplace transform has been established in [12].

We refer to (1.1) as the forward problem. The adjoint to (1.1), which we refer to as the backward problem, is associated with transition probabilities for the solution of a stochastic differential equation with jump returns from the boundary. Further details of this connection may be found in Mansourati's thesis [12]. Because of biorthogonality relations between eigenfunctions of the forward and backward problems, the eigenfunctions of the backward problem figure in the solution of the forward problem. Consequently we study the two problems together.

In the case of the backward problem, there have been investigations of similar problems. The earliest instance appears to be in a paper by Wilder [14]. Of the recent literature, the work of Cole [6], Day [7], and Friedman [10] is close. Friedman's work [10] might initially appear to include some of our results. However, in order to establish existence and uniqueness of a solution, Friedman had to make an assumption on $F_{L}$ and $F_{R}$ which would always be violated when $F_{L}$ and $F_{R}$ are probability distribution functions. We avoid Friedman's condition by using a different method to establish existence and uniqueness.

In a general way, our discussion can be regarded as a development of one of the threads in Feller's classic papers [8, 9]. A recent paper on another type of diffusion process with jump returns to the interior is that of Baccelli and Fayolle [1].

After explaining the connection with birth-death processes in Sec. 2, we establish the existence and uniqueness of solutions of the forward and backward problems in Sec. 3 . In Sec. 4 we deduce some facts about the eigenvalues and eigenfunctions associated with the problem. In Sec. 5 we give two examples and in Sec. 6 we compare some results obtained from the diffusion approximation with exact results for a birth-death process with a finite number of states.

2. Diffusion approximation to a birth-death process. In [3] Campbell treats a birth-death process which is governed by the system of ordinary differential equations

$$
\frac{d p_{k}}{d \tau}=\mu L_{k} p_{1}+\lambda p_{k-1}-(\lambda+\mu) p_{k}+\mu p_{k+1}+\lambda R_{k} p_{m} \quad(k=1, \ldots, m),
$$

where we define $p_{0}(\tau)=p_{m+1}(\tau)=0$. We interpret $p_{k}(\tau)$ as the probability that the system is in state $k(k=1,2, \ldots, m)$ at time $\tau$. Births occur at rate $\lambda$ and deaths occur at rate $\mu$, where $\lambda$ and $\mu$ are positive constants. If the system is in state 1 and 
a death occurs, it moves to state $k$ with probability $L_{k}$, and if the system is in state $m$ and a birth occurs, it moves to state $k$ with probability $R_{k}$. Thus $L_{k} \geq 0, R_{k} \geq 0$, and $\sum L_{k}=\sum R_{k}=1$. It is these boundary conditions at states 1 and $m$ that distinguish this from a conventional birth-death process.

This process could describe an inventory in which the supplier holds a "fire sale" to dispose of stock if the warehouse is full or buys from another supplier when the warehouse is empty. It could describe a buffer at a node in a communications network, where some messages are removed and sent by an alternate route when the buffer fills, and where messages from other buffers are brought in for alternate routing whenever the buffer becomes empty. Other applications are possible.

When the number of states $m$ is large, the system of ordinary differential equations can be approximated by a partial differential equation. Some idea of the goodness of the approximation involved can be obtained from [2], where a special case of (2.1) is solved and the corresponding partial differential equation is also solved. Additional results on this question appear in Sec. 6. For a more general discussion of diffusion approximations, see $[5,11,13]$.

Let $(m+1)^{-1}=\Delta x, x_{k}=k \Delta x, p_{k}(\tau)=\varphi\left(x_{k}, \tau\right) \Delta x$, and define

$$
F_{L}^{m}(x)=\sum_{k=1}^{m} L_{k} H\left(x-x_{k}\right), \quad F_{R}^{m}(x)=\sum_{k=1}^{m} R_{k} H\left(x-x_{k}\right),
$$

where $H$ denotes the unit step function $(H(x)=0$ for $x<0$ and $H(x)=1$ for $x \geq 0)$ so that $F_{L}^{m}$ and $F_{R}^{m}$ are the probability distribution functions corresponding to $\left(L_{1}, \ldots, L_{m}\right)$ and $\left(R_{1}, \ldots, R_{m}\right)$. If we write

$$
\Delta F_{L}^{m}\left(x_{k}\right)=F_{L}^{m}\left(x_{k}\right)-F_{L}^{m}\left(x_{k-1}\right)=L_{k}
$$

and similarly for $\Delta F_{R}^{m}$, and recall that $p_{0}(\tau)=p_{m+1}(\tau)=0,(2.1)$ becomes

$$
\begin{aligned}
\frac{d \varphi}{d \tau}= & \lambda(\Delta x)^{2} \frac{\left[\varphi\left(x_{k+1}, \tau\right)-2 \varphi\left(x_{k}, \tau\right)+\varphi\left(x_{k-1}, \tau\right)\right]}{(\Delta x)^{2}} \\
& -(\lambda-\mu) \Delta x \frac{\left[\varphi\left(x_{k+1}, \tau\right)-\varphi\left(x_{k}, \tau\right)\right]}{\Delta x}+\mu(\Delta x)^{2} \frac{\Delta F_{L}^{m}\left(x_{k}\right)}{\Delta x} \frac{\left[\varphi\left(x_{1}, \tau\right)-\varphi(0, \tau)\right]}{\Delta x} \\
& -\lambda(\Delta x)^{2} \frac{\Delta F_{R}^{m}\left(x_{k}\right)}{\Delta x} \frac{\left[\varphi(1, \tau)-\varphi\left(x_{m}, \tau\right)\right]}{\Delta x} .
\end{aligned}
$$

In the diffusion approximation we replace the difference quotients by derivatives, $\lambda(\Delta x)^{2}$ and $\mu(\Delta x)^{2}$ by $\sigma^{2} / 2$, and $(\lambda-\mu) \Delta x$ by $\beta$. We assume that, as $m \rightarrow \infty, F_{L}^{m}$ and $F_{R}^{m}$ approach limits $F_{L}$ and $F_{R}$ which are absolutely continuous with bounded derivatives $f_{L}$ and $f_{R}$ except at a finite number $N$ (possibly zero) of interior points $a_{i}(i=1,2, \ldots, N)$ where $F_{L}$ jumps by an amount $\rho_{i}$ and $F_{R}$ jumps by an amount $\gamma_{i}$. The diffusion approximation then gives

$$
\begin{aligned}
& \frac{\partial \varphi}{\partial \tau}(x, \tau)=\frac{\sigma^{2}}{2} \frac{\partial^{2} \varphi}{\partial x^{2}}(x, \tau)-\beta \frac{\partial \varphi}{\partial x}(x, \tau) \\
& +\frac{\sigma^{2}}{2}\left[f_{L}(x) \frac{\partial \varphi}{\partial x}(0, \tau)-f_{R}(x) \frac{\partial \varphi}{\partial x}(1, \tau)\right]
\end{aligned}
$$


for all $x \in(0,1)$ except $x=a_{1}, \ldots, a_{x}$. For notational convenience, we define $a_{0}=0$, $a_{N+1}=1$, and assume $a_{i}<a_{i+1}$ for each $i$. Because $p_{()}$and $p_{m+1}$ vanish, we have boundary conditions

$$
\varphi(0, \tau)=\varphi(1, \tau)=0 .
$$

At points where $F_{l}$, or $F_{k}$ is discontinuous, replace $\varphi\left(x_{k+1}, \tau\right)-\varphi\left(x_{k}, \tau\right)$ by

$$
\frac{\partial \varphi}{\partial x}\left(x_{k}, \tau\right) \Delta x
$$

recognize that $\partial \varphi / \partial x$ will be discontinuous there, and rewrite $(2.2)$ as

$$
\begin{aligned}
& \frac{\partial \varphi}{\partial \tau}=\lambda \Delta x {\left[\frac{\partial \varphi}{\partial x}\left(x^{+}, \tau\right)-\frac{\partial \varphi}{\partial x}\left(x^{\cdots}, \tau\right)\right]-(\lambda-\mu) \Delta x \frac{\partial \varphi}{\partial x}\left(x^{+}, \tau\right) } \\
&+\mu \Delta x \Delta F_{l}^{\prime \prime \prime}(x) \frac{\partial \varphi}{\partial x}(0, \tau)-\lambda \Delta x \Delta F_{R}^{\prime \prime \prime}(x) \frac{\partial \varphi}{\partial x}(1, \tau) .
\end{aligned}
$$

Multiply by $\Delta x$, make the above replacements for $\lambda(\Delta x)^{2}$. etc., let $\Delta x \rightarrow 0$, and get at $x=a_{i}$.

$$
\frac{\partial \varphi}{\partial x}\left(a_{i}^{+}, \tau\right)-\frac{\partial \varphi}{\partial x}\left(a_{i}, \tau\right)+\rho_{i} \frac{\partial \varphi}{\partial x}(0, \tau)-\gamma_{i} \frac{\partial \varphi}{\partial x}(1, \tau)=0 .
$$

The forward problem is to find a function $\varphi$ with domain $\{(x, \tau): 0 \leq x \leq 1 . \tau \geq 0\}$ which is such that

(i) $\varphi(x, \tau)$ is a continuous function of $x$ for each $\tau$ and a continuous function of $\tau$ for each $x$,

(ii) on each strip $\left\{(x, \tau): a_{i}<x<a_{i+1}, \tau \geq 0\right\} . i=0,1 \ldots, N . \varphi_{r r}(x, \tau), \varphi, r, r(x, \tau)$. and $\varphi_{\tau}(x, \tau)$ (where subscripts denote partial derivatives) are continuous functions of $x$ for each $\tau$ and of $\tau$ for each $x$.

(iii) $\varphi_{. r}(x, \tau)$ has right and left limits. $\varphi_{, r}\left(a_{i}^{+}, \tau\right)$ and $\varphi_{, r}\left(a_{i}^{-}, \tau\right)$ at $a_{i}$, for $i=1,2, \ldots N$.

(iv) $\varphi_{x}(x, \tau)$ has a right linit $\varphi_{x}(0, \tau)$ at () and a left limit $\varphi_{. r}(1, \tau)$ at 1 ,

(v) $\varphi(x, 0)=\varphi_{0}(x)$. where $\varphi_{01}$ is a given initial function.

(vi) $\varphi(x, \tau)$ satisfies $(2.3)-(2.5)$.

In addition to the forward problem we consider the associated backward problem: to find a function $\Phi$ which, together with the derivatives $\Phi_{r}, \Phi_{r, r}$, and $\Phi_{\tau}$, is continuous on the same domain $\{(x, \tau): 0 \leq x \leq 1, \tau \geq 0\}$, and which satisfies

$$
\begin{aligned}
\frac{\partial \Phi}{\partial \tau} & =\frac{\sigma^{2}}{2} \frac{\partial^{2} \Phi}{\partial x^{2}}+\beta \frac{\partial \Phi}{\partial x}, \\
\Phi(0, \tau) & =\int_{01}^{1} \Phi(\xi, \tau) d F_{L}(\xi), \\
\Phi(1 . \tau) & =\int_{0}^{1} \Phi(\xi, \tau) d F_{R}(\xi) .
\end{aligned}
$$

As was mentioned in the Introduction. boundary conditions like (2.7) and (2.8) have been considered before $[6,7,10,14]$. However. our existence and uniqueness results seem to be new. For a probabilistic interpretation of the backward problem, see Mansouratis thesis [12]. 
Equation (2.2) can be simplified by putting

$$
t=\sigma^{2} \tau / 2, \quad \omega=\beta / \sigma^{2},
$$

and letting

$$
\begin{gathered}
V(x, t)=\exp \left[\omega^{2} t-\omega x\right] \varphi\left(x, \frac{2 t}{\sigma^{2}}\right), \\
f_{0}(x)=e^{\omega x} f_{L}(x), \quad f_{1}(x)=e^{\omega(1-x)} f_{R}(x), \\
p_{i}=e^{-\omega a_{i}} \rho_{i}, \quad q_{i}=e^{\omega\left(1-a_{i}\right)} \gamma_{i}, \\
F_{0}(x)=\int_{0}^{x} e^{-\omega \xi} d F_{L}(\xi), \quad F_{1}(x)=\int_{0}^{x} e^{\omega(1-\xi)} d F_{R}(\xi) .
\end{gathered}
$$

With this notation, the forward problem becomes

$$
\begin{gathered}
\frac{\partial V}{\partial \tau}(x, t)=\frac{\partial^{2} V}{\partial x^{2}}(x, t)+f_{0}(x) \frac{\partial V}{\partial x}(0, t)-f_{1}(x) \frac{\partial V}{\partial x}(1, t) \\
V(0, t)=V(1, t)=0, \\
V(x, 0)=V_{0}(x)
\end{gathered}
$$

and

$$
V_{x}\left(a_{i}^{+}, t\right)-V_{x}\left(a_{i}^{-}, t\right)+p_{i} V_{x}(0, t)-q_{i} V_{x}(1, t)=0,
$$

for $i=1,2, \ldots, N$.

Similarly, we simplify (2.6) by setting

$$
U(x, t)=\exp \left[\omega^{2} t+\omega x\right] \Phi\left(x, \frac{2 t}{\sigma^{2}}\right) .
$$

This gives the new form of the backward problem

$$
\begin{gathered}
\frac{\partial U}{\partial t}=\frac{\partial^{2} U}{\partial x^{2}}, \\
U(0, t)=\int_{0}^{1} U(x, t) d F_{0}(x), \\
U(1, t)=\int_{0}^{1} U(x, t) d F_{1}(x),
\end{gathered}
$$

and

$$
U(x, 0)=U_{0}(x)
$$

Note that, in view of $(2.10)$, when $F_{L}$ and $F_{R}$ are probability distribution functions it is not possible that both inequalities $F_{0}(1)<1$ and $F_{1}(1)<1$ hold, no matter what the sign of $\omega$. This fact prevents us from applying Friedman's [10] existence theorem to our problem.

We sometimes refer to (2.11)-(2.14) and (2.16)-(2.19) as the scaled forward and backward problems respectively. 
3. Existence and uniqueness of solutions. The basic idea that we use to solve either the forward or backward equation is to find the solution first on the boundaries $x=0$ and $x=1$. Once the solution and its derivatives are known on the boundaries, the well-known theory of the one-dimensional heat equation provides the solution everywhere. We treat the scaled problems in this section, beginning with the slightly simpler backward problem (2.16)-(2.19).

If $(2.17)$ and $(2.18)$ are replaced by the conditions

$$
U(0, t)=b_{0}(t), \quad U(1, t)=b_{1}(t),
$$

where $b_{0}$ and $b_{1}$ are continuous, then the unique bounded solution of the heat equation (2.16) satisfying (3.1) and (2.19) is [4]

$$
\begin{array}{rl}
U(x, t)=\int_{0}^{1} & G(x, \xi ; t) U_{0}(\xi) d \xi-2 \int_{0}^{t} \frac{\partial \theta}{\partial x}(x, t-\tau) b_{0}(\tau) d \tau \\
& +2 \int_{0}^{t} \frac{\partial \theta}{\partial x}(x-1, t-\tau) b_{1}(\tau) d \tau
\end{array}
$$

where

$$
\begin{aligned}
G(x, \xi ; t) & =2 \sum_{n=1}^{\infty} e^{-n^{2} \pi^{2} t} \sin (n \pi x) \sin (n \pi \xi) \\
& =\theta(x-\xi, t)-\theta(x+\xi, t), \\
\theta(x, t) & =\sum_{m=-\infty}^{+\infty} K(x+2 m, t), \quad t>0,
\end{aligned}
$$

and

$$
K(x, t)=\frac{1}{\sqrt{4 \pi t}} \exp \left\{-\frac{x^{2}}{4 t}\right\}, \quad t>0 .
$$

In order to obtain equations for $b_{0}$ and $b_{1}$, we integrate (3.2) with respect to $d F_{0}$ and $d F_{1}$ and use (2.17) and (2.18). If $U$, as given by (3.2), is a solution of (2.16)-(2.19), then $b_{0}$ and $b_{1}$ must satisfy the pair of integral equations

$$
b_{i}(t)=\mathcal{G}_{i}(t)+\sum_{j=0}^{1} \int_{0}^{t} \mathcal{N}_{i j}(t-\tau) b_{j}(\tau) d \tau,
$$

for $i=0,1$, where we set

$$
\begin{aligned}
\mathcal{G}_{i}(t) & =\int_{0}^{1}\left(\int_{0}^{1} G(x, \xi ; t) U_{0}(\xi) d \xi\right) d F_{i}(x), \\
\mathcal{N}_{i 0}(t) & =-2 \int_{0}^{1} \frac{\partial \theta}{\partial x}(x-t) d F_{i}(x), \\
\mathcal{N}_{i 1}(t) & =2 \int_{0}^{1} \frac{\partial \theta}{\partial x}(x-1, t) d F_{i}(x) .
\end{aligned}
$$

We show first that the pair of Volterra integral equations $(3.6)$ has a solution $\left(b_{0}, b_{1}\right)$ and then verify that, for this $b_{0}$ and $b_{1}, U$ in (3.2) is the desired solution. Some care is needed because of the singular behavior of $K$ as $t \rightarrow 0$. 
LEMMA 3.1. If $U_{0}$ is bounded and measurable and if $F_{0}$ and $F_{1}$ are absolutely continuous with bounded derivatives $f_{0}$ and $f_{1}$ except at the interior points $a_{1}, a_{2}, \ldots, a_{N}$ where they have finite jumps, then $\mathcal{G}_{i}(i=0,1)$ is continuous for $t>0$ and

$$
\lim _{t \rightarrow 0^{+}} \mathcal{G}_{i}(t)=\int_{0}^{1} U_{0}(x) d F_{i}(x) .
$$

Moreover, if $U_{0}$ is nonnegative, $\mathcal{G}_{i}$ is nonnegative.

Proof. If

$$
h(x, t)=\int_{0}^{1} G(x, \xi ; t) U_{0}(\xi) d \xi
$$

then it is well known [4] that $h$ is continuous for $t>0$ and that

$$
\lim _{t \rightarrow 0^{+}} h(x, t)=U_{0}(x)
$$

Thus (3.7) follows from the dominated convergence theorem. Nonnegativity follows from the fact that $h$, as a solution of the heat equation with nonnegative initial data, is known to be nonnegative.

Lemma 3.2. If $F_{0}$ and $F_{1}$ are as described in Lemma 3.1, then $\mathcal{N}_{i j}$ is continuous for $t>0$ and $t^{1 / 2} \mathcal{N}_{i j}(t)$ is bounded as $t \rightarrow 0^{+}$. Moreover, $\mathcal{N}_{i j}$ is nonnegative.

Proof. Continuity for $t>0$ follows from the uniform convergence of the series (3.4) and a corresponding one for the derivative. For $0 \leq x \leq 1$, it is clear that only one term in the series can be badly behaved as $t \rightarrow 0$. We have

$$
-2 \frac{\partial \theta}{\partial x}(x, t)=\frac{x}{2 \sqrt{\pi} t^{3 / 2}} \exp \left[-x^{2} /(4 t)\right]+R_{0}(x, t)
$$

where $R_{0}$ is the sum of the terms for $m \neq 0$ in the derivative of (3.4). Integration with respect to $d F_{i}(x)$ on $(0,1)$ and the change of variables $u=x / \sqrt{2 t}$ yields

$$
\mathcal{N}_{i 0}(t)=\frac{1}{\sqrt{\pi t}} \int_{0}^{1 / \sqrt{2 t}} u \exp \left[-u^{2} / 2\right] f_{i}(\sqrt{2 t} u) d u+o(t)
$$

as $t \rightarrow 0$. Note that jumps in $F_{i}$ occur at points $a_{i}$ which are bounded away from zero. It follows that contributions from these jumps are $o(t)$ and only the contribution from $f_{i}$ can affect the behavior near $t=0$. Since $f_{i}$ is, by hypothesis, bounded, it follows that $t^{1 / 2} \mathcal{N}_{i 0}(t)$ is bounded as $t \rightarrow 0^{+}$. An exactly similar calculation shows that $t^{1 / 2} \mathcal{N}_{i 1}(t)$ is bounded.

As noted earlier, $G(x, \xi ; t) \geq 0$ for $0 \leq x, \xi \leq 1$. In view of (3.3), this implies that, for $x \in(0,1)$ and $t>0$,

$$
\frac{\partial \theta}{\partial x}(x, t)=\lim _{\xi \rightarrow 0^{+}} \frac{\theta(x+\xi, t)-\theta(x-\xi, t)}{2 \xi} \leq 0,
$$

and from this $\mathcal{N}_{i 0} \geq 0$. Also, by (3.4) and (3.5), $\theta$ is an even function of $x$, so that $\frac{\partial \theta}{\partial x}(x-1, t) \geq 0$ for $x \in(0,1)$. Consequently $\mathcal{N}_{i 1} \geq 0$. 
LEMma 3.3. Under the hypotheses of Lemma 3.1, there exists a unique continuous solution of the pair of integral equations (3.6) for $t>0$. If $U_{0}$ is nonnegative, both $b_{0}$ and $b_{1}$ are nonnegative.

Proof. The proof is almost a standard successive approximation proof, slightly complicated by the mild singularity of the kernel functions. Let

$$
\begin{gathered}
b_{i}^{(n)}(t)=\mathcal{G}_{i}(t)+\sum_{j=0}^{1} \int_{0}^{l} \mathcal{N}_{i j}(t-\tau) b_{j}^{(n-1)}(\tau) d \tau, \\
b_{i}^{(0)}(t)=\mathcal{G}_{i}(t),
\end{gathered}
$$

for $i=0,1$ and $n=1,2, \ldots$ Then

$$
b_{i}^{(n)}(t)=\mathcal{G}_{i}(t)+\sum_{m=1}^{n} \sum_{j=0}^{1} \int_{0}^{l} \mathcal{N}_{i, j}^{(m)}(t-\tau) \mathcal{G}_{j}(\tau) d \tau,
$$

where

$$
\begin{aligned}
\mathcal{N}_{i j}^{(n+1)}(t) & =\sum_{k=0}^{1} \int_{0}^{t} \mathcal{N}_{i k}(t-\tau) \mathcal{N}_{k, j}^{(n)}(\tau) d \tau, \quad n \geq 1, \\
\mathcal{N}_{i j}^{(1)}(t) & =\mathcal{N}_{i, j}(t) .
\end{aligned}
$$

Using Lemmas 3.1 and 3.2, we obtain an estimate

$$
\left|\sum_{j=0}^{1} \int_{0}^{t} \mathcal{N}_{i j}^{(m)}(t-\tau) \mathcal{G}_{j}(\tau) d \tau\right| \leq c\left(\frac{\pi}{t}\right)^{1 / 2} \frac{(2 k \sqrt{t \pi})^{m}}{\Gamma\left(\frac{m+1}{2}\right)},
$$

for $0<t<T$, where

$$
\begin{aligned}
& k=\max _{i, j \in\{0.1\}} \sup _{0<t<T}\left(t^{1 / 2} \mathcal{N}_{i j}(t)\right), \\
& c=\max _{i \in\{0.1\}} \sup _{0<t<T} \mathcal{G}_{i}(t),
\end{aligned}
$$

and $\Gamma$ denotes the gamma function. From (3.10) it follows that the sequence $b_{i}^{(n)}$ of $(3.9)$ converges uniformly on any interval $[0, T]$. Thus there are limits $b_{0}(t)$ and $b_{1}(t)$ that satisfy (3.6). Uniqueness is easily demonstrated. By Lemma $3.2, \mathcal{N}_{i j}^{(m)} \geq 0$ for each $m$, and consequently it is evident from (3.9) and Lemma 3.1 that $b_{0}$ and $b_{1}$ are nonnegative if $U_{0}$ is nonnegative.

THEOREM 3.4. If $U_{0}$ is bounded and measurable and if $F_{0}$ and $F_{1}$ are as described in Lemma 3.1, the scaled backward problem (2.16) (2.19) has a unique solution. If $U_{0}$ is nonnegative, the solution is nonnegative.

Proof. Let $U$ be defined by $(3.2)$ where $\left(b_{0}, b_{1}\right)$ is the solution of $(3.6)$ which exists by Lemma 3.3. By the standard theory of the heat equation, $U$ satisfies (2.16) and (2.19). It is also known [4] that

$$
\lim _{x \rightarrow 0^{+}}-2 \int_{0}^{t} \frac{\partial \theta}{\partial x}(x, t-\tau) b(\tau) d \tau=b(t), \quad \text { for } t>0,
$$


and

$$
\lim _{x \rightarrow 1^{-}}-2 \int_{0}^{t} \frac{\partial \theta}{\partial x}(x, t-\tau) b(\tau) d \tau=0, \quad \text { for } t>0,
$$

and that $G(0, \xi: t)=G(1, \xi: t)=0$. From this we easily obtain

$$
\lim _{x \rightarrow 0^{+}} U(x, t)=b_{0}(t), \quad \lim _{x \rightarrow 1^{-}} U(x, t)=b_{1}(t)
$$

so that (2.17) and (2.18) are also satisfied.

Uniqueness is shown by using Lemma 3.3 to show that $U(0, t)$ and $U(1, t)$ are uniquely defined and then by using the standard theory of the heat equation to show that $U$ is defined elsewhere by (3.2). Nonnegativity when $U_{0}$ is nonnegative follows from the nonnegativity of $b_{0}$ and $b_{1}$ (Lemma 3.3), and from the nonnegativity of $G,-\theta_{x}(x, t)$, and $\theta_{x}(x-1, t)$ (see the proof of Lemma 3.2).

The forward problem. (2.11)-(2.14), is treated in an analogous manner. We discuss those aspects that differ from the earlier treatment. Let

$$
\begin{array}{rl}
V(x, t)=\int_{0}^{1} & G(x, \xi ; t) V_{0}(\xi) d \xi \\
& +\int_{0}^{t} \psi_{0}(\tau)\left[\int_{0}^{1} G(x, \xi ; t-\tau) d F_{0}(\xi)\right] d \tau \\
& +\int_{0}^{t} \psi_{1}(\tau)\left[\int_{0}^{1} G(x, \xi ; t-\tau) d F_{1}(\xi)\right] d \tau \\
= & \int_{0}^{1} \begin{array}{l}
G(x, \xi: t) V_{0}(\xi) d \xi \\
\end{array} \\
& +\int_{0}^{t} \psi_{0}(\tau)\left[\int_{0}^{1} G(x, \xi ; t-\tau) f_{0}(\xi) d \xi+\sum_{i=1}^{N} p_{i} G\left(x, a_{i} ; t-\tau\right)\right] d \tau \\
& \left.\quad \int_{0}^{1} G(x, \xi ; t-\tau) f_{1}(\xi) d \xi+\sum_{i=1}^{N} q_{i} G\left(x, a_{i} ; t-\tau\right)\right] d \tau
\end{array}
$$

where $G$ is defined by (3.3)-(3.5), $V_{0}$ is the initial function in $(2.13)$, and $\psi_{0}$ and $\psi_{1}$ are to be determined so that $(2.11)-(2.14)$ is satisfied.

For $V$ as defined by (3.13), straightforward calculations using the properties of $G$ show that. if $\psi_{0}(t)$ and $\psi_{1}(t)$ are continuous for $t>0$ and are $O\left(t^{-1 / 2}\right)$ as $t \rightarrow 0^{+}$, then

$$
V(0, t)=V(1, t)=0, \quad V\left(x, 0^{+}\right)=V_{0}(x)
$$

and, for $a_{i}<x<a_{i+1}, t>0$,

$$
V_{t}(x, t)=V_{x x}(x, t)+f_{0}(x) \psi_{0}(t)+f_{1}(x) \psi_{1}(t)
$$

Furthermore, for reasons similar to those discussed in the proof of Lemma 3.2, the only terms of (3.13) that contribute to discontinuities of $V_{x}$ at $x=a_{j}$ are those involving 
$G\left(x, a_{j} ; t-\tau\right)$. In fact,

$$
\begin{aligned}
& V_{x}\left(a_{j}^{+}, t\right)-V_{x}\left(a_{j}^{-}, t\right) \\
& \quad=\int_{0}^{t}\left[p_{j} \psi_{0}(\tau)+q_{j} \psi_{1}(\tau)\right]\left[\theta_{x}\left(0^{+}, t-\tau\right)-\theta_{x}\left(0^{-}, t-\tau\right)\right] d \tau \\
& \quad=-\left[p_{j} \psi_{0}(t)+q_{j} \psi_{1}(t)\right]
\end{aligned}
$$

where we have used (3.11) and the fact that $\theta(x, t)$ is even in $x$, so that $\theta_{x}(x, t)$ is odd in $x$. We see that (3.14) and (3.15) become (2.11) and (2.14) if $\psi_{0}(t)=V_{x}(0, t)$ and $\psi_{1}(t)=-V_{x}(1, t)$.

To find equations determining $\psi_{0}$ and $\psi_{1}$, differentiate (3.13) once with respect to $x$ and set $x=0$ and $x=1$. Then $\psi_{0}$ and $\psi_{1}$ must satisfy

$$
\psi_{i}(t)=\mathcal{H}_{i}(t)+\sum_{j=0}^{1} \int_{0}^{t} \mathcal{M}_{i j}(t-\tau) \psi_{j}(\tau) d \tau
$$

where, for $i, j=0,1$,

$$
\mathcal{H}_{i}(t)=(-1)^{i} \int_{0}^{1} G_{x}(i, \xi ; t) V_{0}(\xi) d \xi
$$

and

$$
\mathcal{M}_{i j}(t)=(-1)^{i} \int_{0}^{1} G_{r r}(i, \xi ; t) d F_{j}(\xi) .
$$

Analogously to our earlier discussion, we can prove the following results.

LEMma 3.5. If $V_{0}$ is bounded and measurable, then $\mathcal{H}_{i}$ is continuous for $t>0$ and $t^{1 / 2} \mathcal{H}_{i}(t)$ is bounded as $t \rightarrow 0^{+}$. If $V_{0}$ is nonnegative, then $\mathcal{H}_{i}$ is nonnegative.

LEMma 3.6. If $F_{0}$ and $F_{1}$ are as described in Lemma 3.1, then $\mathcal{M}_{i j}$ is continuous for $t>0$ and $t^{1 / 2} \mathcal{M}_{i j}(t)$ is bounded as $t \rightarrow 0$. Furthermore, $\mathcal{M}_{i j}$ is nonnegative.

LEMMA 3.7. Under the hypotheses of Lemmas 3.5 and 3.6, there exists a unique solution of the pair of integral equations (3.16) which is continuous for $t>0$ and is $O\left(t^{-1 / 2}\right)$ as $t \rightarrow 0$. If $V_{0}$ is nonnegative, both $\psi_{0}$ and $\psi_{1}$ are nonnegative.

The proofs are analogous to the proofs of Lemmas 3.1-3.3 and are omitted. Some further details are available in [12].

THEOREM 3.8. If $V_{0}$ is bounded and measurable and if $F_{0}$ and $F_{1}$ are as described in Lemma 3.1 , the scaled forward problem (2.11)-(2.14) has a unique solution. If $V_{0}$ is nonnegative, the solution is nonnegative.

Proof. Let $V$ be defined by (3.13) where $\left(\psi_{0}, \psi_{1}\right)$ is the solution of (3.16). By the discussion leading up to (3.14) and (3.15), all that we need to establish is that $V_{x}(0, t)=$ $\psi_{0}(t)$ and $V_{x}(1, t)=-\psi_{1}(t)$. But differentiation of (3.13), substitution of $x=0$ and $x=1$, and comparison with the right side of (3.16) shows that this is true. Uniqueness and nonnegativity follow in the same way as in Theorem 3.4.

Clearly, Theorems 3.4 and 3.8 establish existence and uniqueness of solutions $\varphi$ and $\Phi$ of the original forward and backward problems (2.3)-(2.8) with their associated initial conditions. 
4. Eigenvalues and eigenfunctions. Define differential operators $M_{\alpha}$ and $L_{\alpha}$ for each complex number $\alpha$, by

$$
M_{\alpha}[v](x)=v^{\prime \prime}(x)-\alpha^{2} v(x)+f_{0}(x) v^{\prime}(0)-f_{1}(x) v^{\prime}(1)
$$

and

$$
L_{\alpha}[u](x)=u^{\prime \prime}(x)-\bar{\alpha}^{2} u(x),
$$

where the domain of $M_{\alpha}$ is the set

$$
\begin{array}{r}
D=\left\{v: v \text { continuous on }[0,1] ; v^{\prime \prime} \text { continuous on each open interval }\left(a_{i}, a_{i+1}\right)\right. \\
\text { and } v^{\prime} \text { satisfying } v^{\prime}\left(a_{i}^{+}\right)-v^{\prime}\left(a_{i}^{-}\right)=q_{i} v^{\prime}(1)-p_{i} v^{\prime}(0) \text { for } i=1, \ldots, N ; \\
v(0)=v(1)=0\}
\end{array}
$$

and the domain of $L_{\alpha}$ is the set $C^{2}[0,1]$ of functions with continuous second derivatives on $[0,1]$. Define also the functionals $B_{0}$ and $B_{1}$ by

$$
B_{k}[u]=u(k)-\int_{0}^{1} u(x) d F_{k}(x), \quad k=0,1 .
$$

If we attempt to solve the scaled forward problem (2.11)-(2.14) by the method of separating variables or by the Laplace transform method, we are led to the forward eigenvalue problem

$$
M_{\alpha}[v]=0, \quad v \in D .
$$

Similarly, associated with the scaled backward problem (2.16)-(2.19) is the backward eigenvalue problem

$$
L_{\alpha}[u]=0, \quad B_{0}[u]=B_{1}[u]=0 .
$$

In this section we show that the eigenvalues are the same for the two problems, that eigenfunctions corresponding to distinct eigenvalues are biorthogonal, and that for any eigenvalue $\alpha^{2}$, the eigenspaces for the two problems have the same dimension. For the original forward problem, (2.3)-(2.5), we obtain more information about the eigenvalues and the steady-state solution.

We begin with the version of Green's formula given by

LEMMA 4.1. If $v \in D$ and $u \in C^{2}[0,1]$, then

$$
\begin{gathered}
\int_{0}^{1} \overline{u(x)} M_{\alpha}[v](x) d x-\int_{0}^{1} v(x) \overline{L_{\alpha}[u]}(x) d x \\
=v^{\prime}(1) \overline{B_{1}[u]}-v^{\prime}(0) \overline{B_{0}[u]} .
\end{gathered}
$$

The proof follows in the usual way from integration by parts on each interval $\left(a_{i}, a_{i+1}\right)$.

To solve the backward eigenvalue problem, first replace $\alpha$ by $\bar{\alpha}$ and note that the general solution of $L_{\bar{\alpha}}[u]=0$ is

$$
u(x)=A \cosh \alpha x+\frac{B}{\alpha} \sinh \alpha x,
$$


where for $\alpha=0$ we use the limiting value as $\alpha \rightarrow 0$. Application of the conditions $B_{k}[u]=0$ yields

$$
\begin{aligned}
& A B_{0}[\cosh \alpha x]+B B_{0}\left[\alpha^{-1} \sinh \alpha x\right]=0 \\
& A B_{1}[\cosh \alpha x]+B B_{1}\left[\alpha^{-1} \sinh \alpha x\right]=0 .
\end{aligned}
$$

The determinant of $(4.3),(4.4)$ is

$$
\Delta(\alpha)=B_{0}[\cosh \alpha x] B_{1}\left[\alpha^{-1} \sinh \alpha x\right]-B_{0}\left[\alpha^{-1} \sinh \alpha x\right] B_{1}[\cosh \alpha x] .
$$

Thus if $\Delta(\alpha)=0, \alpha^{2}$ is an eigenvalue and (4.2)-(4.4) determine the eigenfunctions. Clearly $\Delta(\alpha)$ is an entire function of $\alpha$; thus it has only a finite number of zeros inside any finite circle. Since $\Delta(-\alpha)=\Delta(\alpha)$ and $\Delta(\bar{\alpha})=\overline{\Delta(\alpha)}, \alpha$ is a zero if and only if $-\alpha$ and $\bar{\alpha}$ are zeros. In particular, eigenvalues $\alpha^{2}$ occur in conjugate pairs.

To solve the forward eigenvalue problem, we use (4.1) to determine the derivatives $v^{\prime}(0)$ and $v^{\prime}(1)$ of an eigenfunction $v$. Once these values are known, we can find $v$ easily. That is, knowing $v(0)=0$ and knowing $v^{\prime}(0)$ and $v^{\prime}(1)$ we can solve $M_{\alpha}[v]=0$ on $\left(0, a_{1}\right)$. Then continuity gives $v\left(a_{1}^{+}\right)=v\left(a_{1}^{-}\right)$and the jump condition gives $v^{\prime}\left(a_{1}^{+}\right)$, once $v^{\prime}\left(a_{1}^{-}\right)$ is known. With these values the differential equation is solved on $\left(a_{1}, a_{2}\right)$, and so on. If $v^{\prime}(0)=v^{\prime}(1)=0$, we obtain the trivial solution. To get the conditions on $v^{\prime}(0)$ and $v^{\prime}(1)$, let $\alpha^{2}$ be an eigenvalue and let $v$ be an eigenfunction. Apply (4.1) twice, once with $u(x)=\cosh \bar{\alpha} x$ and the second time with $u(x)=(\sinh \bar{\alpha} x) / \bar{\alpha}$ (so that in each case $\left.L_{\alpha}[u]=0\right)$, to get

$$
-v^{\prime}(0) B_{0}[\cosh \alpha x]+v^{\prime}(1) B_{1}[\cosh \alpha x]=0
$$

and

$$
-v^{\prime}(0) B_{0}\left[\alpha^{-1} \sinh \alpha x\right]+v^{\prime}(1) B_{1}\left[\alpha^{-1} \sinh \alpha x\right]=0 .
$$

These equations, as equations for $\left(-v^{\prime}(0), v^{\prime}(1)\right)$, are the transposes of (4.3), (4.4). If $\Delta(\alpha) \neq 0$, there is only the trivial solution; if $\Delta(\alpha)=0$ and the rank of the matrix is one, each of (4.3), (4.4) and (4.6), (4.7) has one linearly independent solution, and if the rank is zero, each pair of equations has two linearly independent solutions. Thus we have proved

THEOREM 4.2. The forward and backward eigenvalue problems have the same eigenvalues. If $\alpha^{2}$ is an eigenvalue, the number of linearly independent solutions of the forward problem equals the number of linearly independent solutions of the backward problem.

Biorthogonality of eigenfunctions also follows immediately from (4.1), as follows:

THEOREM 4.3. Let $v$ be an eigenfunction of the forward problem with eigenvalue $\alpha^{2}$ and let $u$ be an eigenfunction of the backward problem with eigenvalue $\beta^{2}$. If $\alpha^{2} \neq \beta^{2}$,

$$
\int_{0}^{1} \bar{u}(x) v(x) d x=0 .
$$

Proof. Since

$$
L_{\alpha}[u]=L_{\beta}[u]+\left(\bar{\beta}^{2}-\bar{\alpha}^{2}\right) u=\left(\bar{\beta}^{2}-\bar{\alpha}^{2}\right) u
$$


and $M_{\alpha}[v]=0$, substitution in (4.1) gives

$$
\left(\alpha^{2}-\beta^{2}\right) \int_{0}^{1} v(x) \bar{u}(x) d x=0,
$$

from which the result follows.

Biorthogonality of the eigenfunctions associated with the original forward and backward problems (2.3)-(2.8), also with weight function one, follows easily from (2.9) and (2.15), or directly from the differential equations (2.3) and (2.6).

For expansion theorems and stability theorems, we often need bounds on eigenvalues. A coarse bound is given by

THEOREM 4.4. Under our general hypotheses about the functions $F_{0}$ and $F_{1}$, the zeros of $\Delta(\alpha)$ all lie in some strip $-a \leq \operatorname{Re}(\alpha) \leq a$ and the eigenvalues $\alpha^{2}$ lie in a half-plane $\operatorname{Re}\left(\alpha^{2}\right) \leq a^{2}$.

Proof. A little algebra shows that

$$
\begin{gathered}
\alpha \Delta(\alpha)=\sinh \alpha-\int_{0}^{1} \sinh \alpha(1-x) d F_{0}(x)-\int_{0}^{1} \sinh \alpha x d F_{1}(x) \\
+\int_{0}^{1} \int_{0}^{1} \sinh \alpha(y-x) d F_{0}(x) d F_{1}(y) .
\end{gathered}
$$

Recall that the jump-points, if any, of $F_{0}$ and $F_{1}$ are bounded away from 0 and 1 , so that the measures $d F_{0}(x)$ and $d F_{1}(x)$ are of the form $f_{0}(x) d x$ and $f_{1}(x) d x$, with bounded $f_{0}$ and $f_{1}$ near the values $x=0$ and $x=1$. Thus, if we let $\alpha=\alpha_{1}+i \alpha_{2}$,

$$
\lim _{\alpha_{1} \rightarrow \infty}\left[2 e^{-\alpha} \alpha \Delta(\alpha)\right]=1
$$

Consequently there is a positive number $a$ such that $\Delta(\alpha) \neq 0$ for $\alpha_{1}>a$. Since $\Delta(-\alpha)=\Delta(\alpha)$, it follows that $\Delta(\alpha) \neq 0$ for $\alpha_{1}<-a$, and the zeros lie in the strip $-a \leq \alpha_{1} \leq a$.

Theorem 4.4 holds whenever $F_{0}$ and $F_{1}$ have the properties listed in Lemma 3.1, i.e., they are absolutely continuous with bounded derivatives except at a finite number of points in the open interval $(0,1)$, where they may have finite jumps. However, when they are also related to probability distribution functions by $(2.10)$, more precise information is available. In this case it is more useful to deal with the unscaled equations.

Theorem 4.5. Consider the following eigenvalue problem associated with the backward problem $(2.6)-(2.8)$ on $[0,1]$ where $F_{L}$ and $F_{R}$ are probability distribution functions:

$$
\begin{gathered}
\frac{\sigma^{2}}{2} \Phi^{\prime \prime}(x)+\beta \Phi^{\prime}(x)=\alpha^{2} \Phi(x), \\
\Phi(0)=\int_{0}^{1} \Phi(x) d F_{L}(x) \\
\Phi(1)=\int_{0}^{1} \Phi(x) d F_{R}(x) .
\end{gathered}
$$


Zero is an eigenvalue associated with the constant eigenfunction and, if $\alpha^{2}$ is any eigenvalue, $\operatorname{Re}\left(\alpha^{2}\right) \leq 0$.

Proof. For $\alpha^{2}=0$, direct calculations using the fact that

$$
\int_{0}^{1} d F_{L}(x)=\int_{0}^{1} d F_{R}(x)=1
$$

show that the only solution of (4.8) is the constant function.

Next, given an arbitrary eigenfunction $\Phi$, we show that $|\Phi|$ has a maximum at some interior point $c$ in the interval $(0,1)$. Since $|\Phi|$ is continuous on $[0,1]$, it reaches its maximum on $[0,1]$. If the maximum is achieved only at 0 or 1 , or both, then $|\Phi(x)|<$ $|\Phi(0)|$ or $|\Phi(x)|<|\Phi(1)|$, or both, for all $x \in(0,1)$. Assuming that the first inequality holds, we have the following contradiction:

$$
\begin{aligned}
|\Phi(0)|=\left|\int_{0}^{1} \Phi(\xi) d F_{L}(\xi)\right| & \leq \int_{(0.1)}|\Phi(\xi)| d F_{L}(\xi) \\
& <\int_{(0.1)}|\Phi(0)| d F_{L}(\xi)=|\Phi(0)| .
\end{aligned}
$$

The strict inequality follows from the assumption that $F_{L}$ has jump discontinuities at interior points only. A similar contradiction may be deduced from the inequality involving 1. Therefore, there exists an interior point $c$ where $|\Phi|$ reaches its maximum.

Now, multiplying (4.8a) by the complex conjugate $\bar{\Phi}$ implies that

$$
\frac{\sigma^{2}}{2}\left[\bar{\Phi} \Phi^{\prime \prime}+\left|\Phi^{\prime}\right|^{2}\right]-\frac{\sigma^{2}}{2}\left|\Phi^{\prime}\right|^{2}+\beta \bar{\Phi} \Phi^{\prime}=\alpha^{2}|\Phi|^{2} .
$$

If $\Phi$ satisfies (4.8a) and is constant, the first and second derivatives would be zero and, consequently, $\alpha^{2} \Phi(x)=0$. If $\alpha \neq 0$, then $\Phi \equiv 0$ which, by definition, cannot be an eigenfunction. Thus, there are no constant eigenfunctions associated with nonzero eigenvalues. Now, at $x=c$, we have

$$
|\Phi(c)|^{2}>0, \quad \frac{d}{d x}|\Phi|^{2}=0, \quad \text { and } \quad \frac{d^{2}}{d x^{2}}|\Phi|^{2} \leq 0 .
$$

Since

$$
\frac{d}{d x}|\Phi(x)|^{2}=2\left[\operatorname{Re}\left(\bar{\Phi} \Phi^{\prime}\right)\right](x)
$$

we have $\left[\operatorname{Re}\left(\bar{\Phi} \Phi^{\prime}\right)\right](c)=0$. Moreover, since

$$
\frac{d^{2}}{d x^{2}}|\Phi(x)|^{2}=2 \operatorname{Re}\left[\bar{\Phi} \Phi^{\prime \prime}+\left|\Phi^{\prime}\right|^{2}\right](x)
$$

we have $\operatorname{Re}\left[\bar{\Phi} \Phi^{\prime \prime}+\left|\Phi^{\prime}\right|^{2}\right](c) \leq 0$. Thus, taking the real part of $(4.9)$ at $x=c$ implies that $\operatorname{Re}\left(\alpha^{2}\right) \leq 0$.

Since zero is an eigenvalue for the backward problem, it is also an eigenvalue of the forward problem. The corresponding eigenfunction, called the steady-state solution, is 
often important in applications. A steady-state solution is a continuous function $g$ on the interval $[0,1]$ that vanishes at the end-points and that satisfies

$$
g^{\prime \prime}(x)-2 \omega g^{\prime}(x)+\left[f_{L}(x) g^{\prime}(0)-f_{R}(x) g^{\prime}(1)\right]=0
$$

on each open interval $\left(a_{i}, a_{i+1}\right)$ for $i=0,1, \ldots, N$, and that satisfies

$$
g^{\prime}\left(a_{i}^{+}\right)-g^{\prime}\left(a_{i}^{-}\right)+\rho_{i} g^{\prime}(0)-\gamma_{i} g^{\prime}(1)=0
$$

for $i=1,2, \ldots, N$, where $\omega=\beta / \sigma^{2}$.

TheOREM 4.6. Define functions $L$ and $R$ by

$$
L(x)=\int_{0}^{x} e^{2 \omega(x-\xi)}\left[1-F_{L}(\xi)\right] d \xi
$$

and

$$
R(x)=\int_{0}^{x} e^{2 \omega(x-\xi)} F_{R}(\xi) d \xi
$$

Then $g_{0}$, defined by

$$
g_{0}(x)=L(x) R(1)-L(1) R(x),
$$

is a steady-state solution. Moreover, $g_{0}(x) \geq 0$ for each $x$ in $[0,1]$.

Proof. It is straightforward to verify that $g_{0}$ satisfies (4.10). Also,

$$
L(x) R(y)-L(y) R(x)=\int_{0}^{y} d \eta \int_{0}^{x} d \xi e^{2 \omega(x+y-\xi-\eta)}\left[Q(\xi) F_{R}(\eta)-Q(\eta) F_{R}(\xi)\right]
$$

where

$$
Q(x)=1-F_{L}(x)
$$

Using the antisymmetry of the integrand about the line $\eta=\xi$, we have, for $y>x$,

$$
L(x) R(y)-L(y) R(x)=\int_{x}^{y} d \eta \int_{0}^{x} d \xi e^{2 \omega(x+y-\xi-\eta)}\left[Q(\xi) F_{R}(\eta)-Q(\eta) F_{R}(\xi)\right]
$$

Since, for $\eta>\xi, Q(\xi)>Q(\eta)$ and $F_{R}(\eta)>F_{R}(\xi)$, the integrand is positive. Hence the integral is positive for $y>x$. The particular case $y=1$ yields the result $g_{0}(x) \geq 0$.

Positivity of $g_{0}(x)$ means that $g_{0}$ can be normalized and converted into a probability density function.

5. Examples. To illustrate the theory, we discuss two examples briefly. The first example corresponds to the diffusion problem where $F_{L}$ and $F_{R}$ each concentrate their masses at $x=1 / 2$. In this example the birth rate is not necessarily equal to the death rate; so we do not assume that $\omega=\beta / \sigma^{2}=0$. When $\omega=0$ we have the case treated in [2]. The second example corresponds to uniform distributions on $[0,1]$ with no jumps and with $\omega=0$. 
ExAmple 1. Let $F_{L}(x)=H\left(x-\frac{1}{2}\right)$ and $F_{R}(x)=H\left(x-\frac{1}{2}\right)$, where $H$ denotes the unit step function as before. Then the eigenfunction problem associated with the original forward problem (2.3)-(2.5) is

$$
\begin{gathered}
\frac{\sigma^{2}}{2}\left[\varphi^{\prime \prime}(x)-2 \omega \varphi^{\prime}(x)\right]=\alpha^{2} \varphi(x), \\
\varphi^{\prime}\left(\frac{1}{2}+\right)-\varphi^{\prime}\left(\frac{1}{2}-\right)=\varphi^{\prime}(1)-\varphi^{\prime}(0), \\
\varphi(0)=\varphi(1)=0,
\end{gathered}
$$

while the eigenfunction problem for the backward problem $(2.6)-(2.8)$ is

$$
\begin{gathered}
\frac{\sigma^{2}}{2}\left[\Phi^{\prime \prime}(x)+2 \omega \Phi^{\prime}(x)\right]=\alpha^{2} \Phi(x), \\
\Phi(0)=\Phi\left(\frac{1}{2}\right)=\Phi(1) .
\end{gathered}
$$

If $\omega \neq 0$, the eigenvalues are simple. It can be verified that the eigenvalues and eigenfunctions separate naturally into two sets, which we label with superscripts (1) and (2), as follows:

$$
\begin{gathered}
\left(\alpha_{n}^{(1)}\right)^{2}=\frac{\sigma^{2}}{2}\left(-4 \pi^{2} n^{2}-\omega^{2}\right) \quad(n=1,2, \ldots), \\
\varphi_{n}^{(1)}(x)= \begin{cases}e^{\omega x}\left[1-(-1)^{n} e^{-\omega / 2}\right] \sin 2 \pi n x, & 0 \leq x \leq \frac{1}{2}, \\
e^{\omega(x-1)}\left[1-(-1)^{n} e^{\omega / 2}\right] \sin 2 \pi n x, & \frac{1}{2} \leq x<1,\end{cases} \\
\Phi_{n}^{(1)}(x)=e^{-\omega x} \sin 2 \pi n x,
\end{gathered}
$$

and

$$
\begin{gathered}
\left(\alpha_{n}^{(2)}\right)^{2}=\left(-8 \pi^{2} n^{2}-4 \pi n \omega i\right) \sigma^{2} \quad(n=0, \pm 1, \pm 2, \ldots), \\
\varphi_{n}^{(2)}(x)= \begin{cases}e^{\omega x} \sinh (\omega-4 \pi n i) x, & 0 \leq x \leq \frac{1}{2}, \\
e^{\omega x} \sinh (\omega-4 \pi n i)(1-x), & \frac{1}{2} \leq x \leq 1, \\
\Phi_{n}^{(2)}(x)=e^{-i 4 n \pi x}\end{cases}
\end{gathered}
$$

As $\omega \rightarrow 0$, the eigenvalues $\left(\alpha_{n}^{(2)}\right)^{2}$ and $\left(\alpha_{-n}^{(2)}\right)^{2}$ merge with $\left(\alpha_{2 n}^{(1)}\right)^{2}$. The end result for $\omega=0$ is the two sets of eigenvalues and eigenfunctions:

$$
\begin{gathered}
\left(\alpha_{n}^{(1)}\right)^{2}=-2 \pi^{2}(2 n+1)^{2} \sigma^{2} \quad(n=1,2, \ldots), \\
\varphi_{n}^{(1)}(x)=\sin 2(2 n+1) \pi x, \\
\Phi_{n}^{(1)}(x)=\sin 2(2 n+1) \pi x,
\end{gathered}
$$


and

$$
\begin{gathered}
\left(\alpha_{0}^{(2)}\right)^{2}=0, \\
\varphi_{0}^{(2)}(x)= \begin{cases}x, & 0 \leq x \leq \frac{1}{2}, \\
1-x, & \frac{1}{2} \leq x \leq 1,\end{cases} \\
\Phi_{0}^{(2)}(x)=1, \\
\left(\alpha_{n}^{(2)}\right)^{2}=-8 n^{2} \pi^{2} \sigma^{2} \quad(n=1,2, \ldots), \\
\varphi_{n}^{(2,1)}(x)= \begin{cases}\sin 4 n \pi x, & 0 \leq x \leq \frac{1}{2}, \\
0, & \frac{1}{2} \leq x \leq 1,\end{cases} \\
\varphi_{n}^{(2,2)}(x)= \begin{cases}0, & 0 \leq x \leq \frac{1}{2}, \\
\sin 4 n \pi x, & \frac{1}{2} \leq x \leq 1,\end{cases} \\
\Phi_{n}^{(2,1)}(x)=\sin 4 n \pi x, \\
\Phi_{n}^{(2,2)}(x)=\cos 4 n \pi x .
\end{gathered}
$$

Thus the eigenspaces corresponding to eigenvalues $\left(\alpha_{n}^{(2)}\right)^{2}, n=1,2, \ldots$, have dimension 2 .

EXAMPLE 2. Let $F_{L}(x)=F_{R}(x)=x$ and let $\omega=0$. Then $F_{0}=F_{L}, F_{1}=F_{R}$, and we can use (4.2)-(4.7). From (4.5) the eigenvalues are zeros of

$$
\Delta(\alpha)=\frac{2}{\alpha} \sinh \frac{\alpha}{2}\left(\cosh \frac{\alpha}{2}-\frac{2}{\alpha} \sinh \frac{\alpha}{2}\right)
$$

multiplied by $\sigma^{2} / 2$. The eigenvalues and eigenfunctions again separate into two sets:

$$
\begin{aligned}
\left(\alpha_{n}^{(1)}\right)^{2}= & -2 n^{2} \pi^{2} \sigma^{2} \quad(n=1,2, \ldots), \\
& \varphi_{n}^{(1)}(x)=\sin 2 n \pi x \\
& \Phi_{n}^{(1)}(x)=\sin 2 n \pi x
\end{aligned}
$$

and

$$
\left(\alpha_{n}^{(2)}\right)^{2}=-\left(\beta_{n}\right)^{2} \sigma^{2} / 2 \quad(n=0,1,2, \ldots),
$$

where $\beta_{0}, \beta_{1}, \beta_{2}, \ldots$ are the nonnegative solutions of

$$
\tan \frac{\beta}{2}=\frac{\beta}{2}
$$

arranged in increasing order with $\beta_{0}=0$,

$$
\begin{aligned}
& \varphi_{0}^{(2)}(x)=x(1-x) \\
& \Phi_{0}^{(2)}(x)=1 \\
& \varphi_{n}^{(2)}(x)=\sin \left(\beta_{n} x / 2\right) \sin \left(\beta_{n}[1-x] / 2\right) \quad(n=1,2, \ldots), \\
& \Phi_{n}^{(2)}(x)=\cos \beta_{n}\left(x-\frac{1}{2}\right) \quad(n=1,2, \ldots) .
\end{aligned}
$$


6. Accuracy of diffusion approximation. One motivation for studying these equations was that they represent, in some sense, an approximation to (2.1) when the number of states is large. In this section we compare the steady-state solution $g_{0}(x)$ given by (4.13) with the steady-state solution of (2.1). We also compare the eigenvalues and eigenfunctions of Example 1, Sec. 5, with the eigenvalues and eigenvectors of the appropriate special case of (2.1). The comparisons provide some evidence that, in cases where (2.1) cannot be solved explicitly, solutions of $(2.3)-(2.5)$ can be used to give good approximations.

(a) Steady-state solutions. To begin, note that integration by parts in (4.11) and (4.12) yields the expressions

$$
2 \omega L(x)=e^{2 \omega x}-1-\int_{0}^{x}\left[e^{2 \omega(x-\xi)}-1\right] d F_{L}(\xi)
$$

and

$$
2 \omega R(x)=\int_{0}^{x}\left[e^{2 \omega(x-\xi)}-1\right] d F_{R}(\xi),
$$

where we assume $\omega \neq 0$. Now replace $F_{L}$ and $F_{R}$ in (6.1) and (6.2) by $F_{L}^{m}$ and $F_{R}^{m}$, the discrete distribution functions which are used in (2.2), and denote the resulting functions by $L^{m}$ and $R^{m}$, so that

$$
2 \omega L^{m}(x)=e^{2 \omega x}-1-\sum_{\left\{\xi_{j} \leq x\right\}} L_{j}\left[e^{2 \omega\left(x-\xi_{j}\right)}-1\right]
$$

and

$$
2 \omega R^{m}(x)=\sum_{\left\{\xi_{j} \leq x\right\}} R_{j}\left[e^{2 \omega\left(x-\xi_{j}\right)}-1\right] .
$$

Now write $\xi_{j}=j /(m+1)$, let $x \rightarrow x_{k}=k /(m+1)$ from the left, and define

$$
w=\frac{\omega}{m+1} \text {. }
$$

Then

$$
2 \omega L^{m}\left(x_{k}^{-}\right)=e^{2 k w}-1-\sum_{j=1}^{k-1} L_{j}\left[e^{2(k-j) w}-1\right]
$$

and

$$
2 \omega R^{m}\left(x_{k}^{-}\right)=\sum_{j=1}^{k-1} R_{j}\left[e^{2(k-j) w}-1\right]
$$

where $k \in\{0,1, \ldots, m+1\}$ and empty sums are defined to be zero. Corresponding to (4.13), we put

$$
g_{0}^{m}\left(x_{k}^{-}\right)=L^{m}\left(x_{k}^{-}\right) R^{m}(1)-L^{m}(1) R^{m}\left(x_{k}^{-}\right) .
$$

To complete the correspondence between steady-state solutions of (2.1) and (2.3)-(2.5) we recall that our assumptions about $\lambda$ and $\mu$ could be written in the form

$$
\lim _{m \rightarrow \infty} \frac{\lambda(m)}{(m+1)^{2}}=\frac{\sigma^{2}}{2}
$$


and

$$
\lim _{m \rightarrow \infty} \frac{\lambda(m)-\mu(m)}{m+1}=\beta
$$

From this, a standard calculation shows that

$$
\lim _{m \rightarrow \infty}\left[\frac{\lambda(m)}{\mu(m)}\right]^{m+1}=e^{2 \omega}
$$

so that, for large $m$,

$$
\frac{\lambda}{\mu} \approx e^{2 \omega /(m+1)}=e^{2 w}
$$

where $w$ is defined by $(6.5)$.

If we replace $e^{2 w}$ by $(\lambda / \mu)$ in (6.6)-(6.8) and do the necessary algebraic simplifications, we find that the resulting expression for $g_{0}^{m}\left(x_{k}^{-}\right)$is just the same (apart from a normalizing constant and necessary adjustments to notation) as the component $\pi_{k}$ of the steady-state solution of (2.1) which is to be found in [3, Eq. (13)]. Thus, replacement of $F_{L}$ and $F_{R}$ by the corresponding discrete distribution functions, replacement of $e^{2 \omega /(m+1)}$ by $\lambda / \mu$, and evaluation of $g_{0}(x)$ at points $k /(m+1), k=0,1, \ldots, m+1$, give the corresponding steady-state solution in the discrete case.

(b) Example 1 and the corresponding discrete case. We consider the special case of (2.1) where the number of states $m$ is replaced by $2 N+1$, where $N$ is an even number, and where $L_{N}=R_{N}=1$, with the other $L_{k}$ and $R_{k}$ equal to zero. This means that returns are always to the midpoint, just as in the diffusion of Example 1. The solution of this problem is given in [2]. As in Example 1 the eigenvalues and eigenvectors divide naturally into two sets. There are $N$ eigenvalues

$$
r_{n}^{(1)}=-\lambda-\mu+2 \sqrt{\lambda \mu} \cos \frac{\pi n}{N+1} \quad(n=1,2, \ldots, N),
$$

and $N+1$ eigenvalues

$$
r_{n}^{(2)}=-2(\lambda+\mu) \sin ^{2} \frac{\pi n}{N+1}-i(\lambda-\mu) \sin \frac{2 \pi n}{N+1} \quad\left(n=0, \pm 1, \ldots, \pm \frac{N}{2}\right) .
$$

For $n \ll N$ we approximate the sine and cosine by Taylor series to order $(N+1)^{-2}$, getting

$$
r_{n}^{(1)} \approx-(\sqrt{\lambda}-\sqrt{\mu})^{2}-\sqrt{\lambda \mu} \frac{\pi^{2} n^{2}}{(N+1)^{2}}
$$

and

$$
r_{n}^{(2)} \approx-2(\lambda+\mu) \frac{\pi^{2} n^{2}}{(N+1)^{2}}-i(\lambda-\mu) \frac{2 \pi n}{N+1} .
$$

For large $N$, the approximations for $\lambda$ and $\mu$ imply that

$$
\lambda \approx \mu \approx \sqrt{\lambda \mu} \approx 2 \sigma^{2}(N+1)^{2}, \quad \lambda-\mu \approx 2 \beta(N+1),
$$

and, after a little algebra, that

$$
(\sqrt{\lambda}-\sqrt{\mu})^{2} \approx \frac{\sigma^{2}}{2} \omega^{2} .
$$


With these replacements, $r_{n}^{(1)}$ and $r_{n}^{(2)}$ become the same as the eigenvalues $\left(\alpha_{n}^{(1)}\right)^{2}$ and $\left(\alpha_{n}^{(2)}\right)^{2}$ of Example 1. Thus, the diffusion approximation gives good estimates of the lower (i.e., $n \ll N$ ) eigenvalues.

The comparison of eigenvectors resembles the comparison that was done for the steadystate solutions. We provide some detail for the first $N$ components of the eigenvectors corresponding to $r_{n}^{(1)}$. The situation for the other $N+1$ components and for the eigenvectors corresponding to $r_{n}^{(2)}$ is essentially the same. The first $N$ components of the right eigenvector are [2, Eq. (11)]

$$
\varphi_{n}(k)=e^{-(N+1-k) w}\left[(-1)^{n}-e^{(N+1) w}\right] \sin \frac{k \pi n}{N+1}, \quad k=1, \ldots, N,
$$

while the $2 N+1$ components of the left eigenvector are [2, Eq. (12)]

$$
\Phi_{n}(k)=(-1)^{n} e^{(N+1-k) w} \sin \frac{k \pi n}{N+1},
$$

where $w=\frac{1}{2} \ln (\lambda / \mu)$. If we now write $x_{k}$ for $k /(2(N+1))$ and, for the reasons given earlier in this section, replace $w$ by $\omega /(2(N+1))$, we get

$$
\varphi_{n}(k)=-\varphi_{n}^{(1)}\left(x_{k}\right), \quad 0<x_{k}<\frac{1}{2}
$$

and

$$
\Phi_{n}(k)=(-1)^{n} e^{\omega / 2} \Phi_{n}^{(1)}\left(x_{k}\right), \quad 0<x_{k}<1 .
$$

Thus, evaluation of the eigenfunctions $\varphi_{n}$ and $\Phi_{n}$ at the points $x_{k}=k /(2(N+1))$ yields, up to multiplying constants, the components of the right and left eigenvectors respectively in [2]. Unlike the situation with eigenvalues, this result does not depend on any assumption that $n \ll N$.

\section{REFERENCES}

[1] F. Baccelli and G. Fayolle, Analysis of models reducible to a class of diffusion processes in the positive quarter plane, SIAM J. Appl. Math. 47, 1367-1385 (1987)

[2] P. G. Buckholtz, L. L. Campbell, R. D. Milbourne, and M. T. Wasan, Analysis of transient behaviour of certain processes with return to a central state, J. Appl. Probab. 20, 61-70 (1983)

[3] L. L. Campbell, Transient analysis of birth-death processes with two boundaries, Canad. J. Stat. 13, 123-130 (1985)

[4] J. R. Cannon, The one-dimensional heat equation, Encyclopedia of Mathematics and its Appl., vol. 23, Addison-Wesley, Reading, MA, 1984

[5] S. Chaimsiri and M. S. Leonard, A diffusion approximation for bulk queues, Management Sci. 27, 1188-1199 (1981)

[6] R. H. Cole, General boundary conditions for an ordinary linear differential system, Trans. Amer. Math. Soc. 111, 521-550 (1964)

[7] W. A. Day, A decreasing property of solutions of parabolic equations with applications to thermoelasticity, Quart. Appl. Math. 41, 468-475 (1983)

[8] W. Feller, The parabolic differential equations and the associated semi-group of transformations, Ann. Math. 55, 468-519 (1952)

[9] W. Feller, Diffusion processes in one dimension, Trans. Amer. Math. Soc. 77, 1-31 (1954)

[10] A. Friedman, Monotonic decay of solutions of parabolic equations with nonlocal boundary conditions, Quart. Appl. Math. 44, 401-407 (1986) 
[11] P. W. Glynn, Diffusion approximations, in Stochastic Models, Chapter 4, edited by D. P. Heyman and M. J. Sobel, North-Holland, New York, 1990

[12] Z. G. Mansourati, Non-classical diffusion equations related to a class of birth-death processes with two boundaries, Ph.D. Thesis, Queen's University at Kingston, January, 1990

[13] G. Pujolle, Réseaux de files d'attente: Méthode des diffusions, Editions Hommes et Techniques, Paris, 1980, pp. 41-72

[14] C. E. Wilder, Expansion problems of ordinary linear differential equations with auxiliary conditions at more than two points, Trans. Amer. Math. Soc. 18, 415-442 (1917) 